\title{
Artigo Visão: Processamento de Banco de Dados em Memória
}

\author{
Tiago R. Kepe ${ }^{1,2}$, Eduardo C. Almeida ${ }^{1}$, Marco A. Z. Alves ${ }^{1}$ \\ ${ }^{1}$ Universidade Federal do Paraná (UFPR) \\ ${ }^{2}$ Instituto Federal do Paraná (IFPR) \\ \{trkepe, eduardo, mazalves\}@inf.ufpr.br
}

\begin{abstract}
Neste artigo, apresentamos nossa visão de como os Sistemas Gerenciadores de Bancos de Dados (SGBD) podem integrar Processamento-emMemória (PIM) em processamento de consultas. PIM promete mitigar os problemas clássicos de "memory-wall" $e$ "energy-wall" presentes nas arquiteturas de computadores que são amplificados pela movimentação de dados na hierarquia de memória por aplicações de análise de dados. Compartilhamos com a comunidade uma análise empírica dos prós e contras do uso de PIM em três principais operadores da álgebra relacional: seleção, projeção e junção. Com base nos resultados obtidos começamos a desenvolver um escalonador de consultas PIM-consciente que apresenta resultados promissores chegando a reduzir em $3 \times$ o tempo de execução de consultas e o consumo de energia em pelo menos $25 \%$. Concluímos nossa visão com uma discussão sobre os desafios e oportunidades para impulsionar pesquisas na concepção de um SGBD com PIM.
\end{abstract}

\section{Introdução}

PIM foi originalmente concebido no final da década de 60 [Kautz 1969] e consiste em adicionar unidades de processamento dentro do dispositivo de memória para uso eficiente da largura de banda interna. Ao longo dos anos, a ideia de PIM foi revisitada de acordo com o progresso das tecnologias de memória. Por exemplo, componentes de processamento foram adicionados em discos magnéticos para executar certos algoritmos de processamento de consultas [Keeton and et al. 1998]. O mesmo ocorreu às tecnologias de memória RAM [Patterson and et al. 1997] que tentaram adicionar unidades lógicas dentro da DRAM para realizar computações específicas. Infelizmente, produtos comerciais não adotaram essas abordagens devido às limitações tecnológicas de hardware e, porque o crescimento contínuo de desempenho de CPU obedecia à lei de Moore e o escalonamento de Dennard. Soluções em discos flash também tentaram embutir unidades funcionais [Do et al. 2013]. Porém, essas soluções não foram exitosas devido à falta de uma interface de programação genérica e o custoso tratamento de erros de hardware.

Sistemas centrados em dados movem grandes quantidades de dados pela hierarquia de memória até a CPU. Por isso, novas arquiteturas PIM emergem devido à introdução das vias de silício em memória (Through-Silicon Vias (TSVs)) que tornaram as memórias 3D viáveis: 3D consiste na integração de chips de DRAM e células lógicas no mesmo dispositivo. Na prática, as memórias 3D invertem o modelo de processamento tradicional, i.e., elas movem a computação até os dados. Atualmente, as GPUs comerciais embutem memórias 3D nas tecnologias: Cubo Híbrido de Memória (HMC) [Jeddeloh and Keeth 2012] e Memória de Alta Largura de Banda (HBM) [Kim and Kim 2014].

Portanto, PIM surge como uma abordagem atrativa para reduzir a movimentação de dados em sistemas centrados em dados. Logo, em nossa visão, já é tempo de discutir 
como SGBDs podem odotar PIM no processamento de consultas. Os maiores benefícios a serem explorados são a redução drástica no consumo de energia e a alta largura de banda interna dos novos dispositivos, pois estes fornecem um alto nível de acesso paralelo aos dados aliado ao processamento em memória. Atualmente, PIM tem sido usado de forma isolada como acelerador de operadores, tais como: seleção [Tome and et al. 2018] e junção [Mirzadeh et al. 2015]. Porém, notamos que o comportamento de cada operador depende de características do conjunto de dados e das configurações do sistema de caches. Neste artigo discutimos os seguintes aspectos para integração de PIM em SGBDs:

I. Idealizamos um processador de consulta PIM-consciente composto por otimizador e escalonador PIM-conscientes para usufruir do paralelismo intra-consulta entre CPU e PIM, e responder a pergunta de pesquisa: Quando é melhor processar os dados no dispositivo de memória ao invés de movê-los pela hierarquia de memória até a CPU?

II. Introduzimos um escalonador PIM-consciente como componente preliminar do processador de consulta que visa intercalar a execução intra-consulta entre CPU e PIM para explorar o potencial de cada dispositivo de processamento.

III. Divulgamos resultados preliminares sobre o impacto de PIM no processamento tradicional de consulta e discutimos a economia de energia com a redução drástica no tempo de execução além de uma ordem de magnitude para certos operadores.

IV. Compartilhamos uma lista de desafios e oportunidades para o desenvolvimento e integração de um SGBD-PIM.

\section{Fundamentos de Arquiteturas PIM}

As memórias 3D emergentes integram quatro ou oito chips de DRAM empilhados e interconectados via TSV até uma camada lógica na base. A pilha de chips de DRAM é organizada de forma vertical, formando partições chamadas "vaults" (Figura 1). As memórias $3 \mathrm{D}$ atuais possuem 32 vaults interdependentes para acessar e processar dados em paralelo alavancando a largura de banda interna em até $320 \mathrm{~GB} / \mathrm{s}$, e suportam instruções aritméticas, lógicas e binárias com operandos de até 16-bytes.

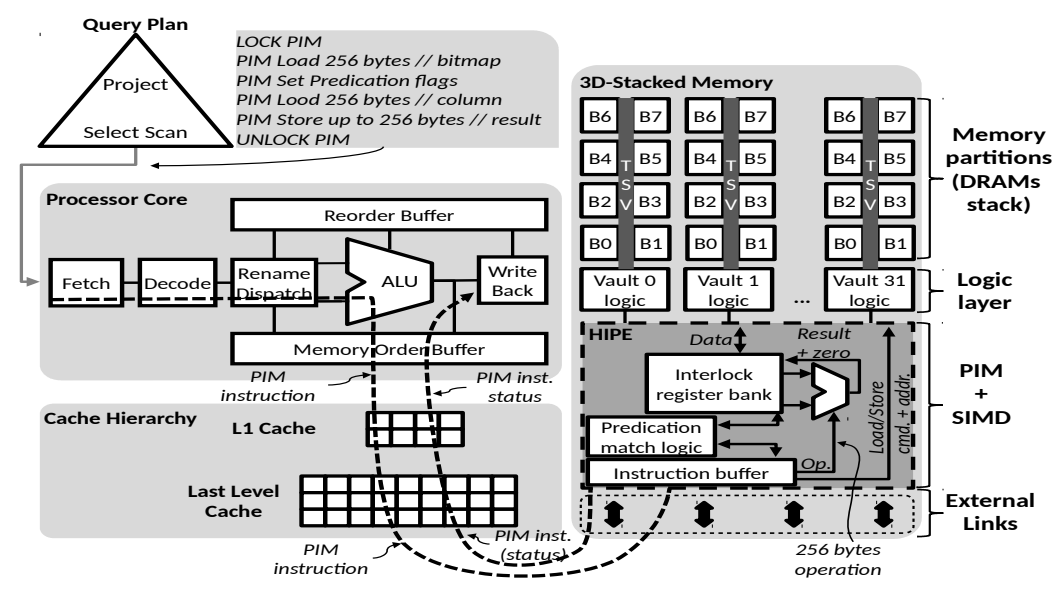

Figura 1. 0 fluxo de uma consulta na arquitetura von Neumann tradicional em conjunto com uma memória 3D com PIM e suporte SIMD.

O trabalho recente de [Tome and et al. 2018] apresenta uma extensão da arquitetura desses dispositivos para operar com instruções SIMD (Single Instruction, Multiple 
Data) em vetores de 256-bytes para processamento de consultas, pois cada vault possui um buffer de 256-bytes para acessar os bancos de memória correspondentes a sua partição. A Figura 1, lado direito, ilustra essa arquitetura PIM com suporte SIMD e, no lado esquerdo, a arquitetura von Neumann tradicional, i.e., o processador com a hierarquia de caches separados. No topo da Figura 1, o processador dispara instruções PIM diretamente ao dispositivo PIM contornando a hierarquia de caches. No final, o dispositivo PIM retorna somente o status da instrução à CPU para continuar a execução do pipeline. Esse mecanismo é a principal vantagem sobre atuais SGBDs (e.g. Netezza e Exasol) que também filtram dados em hardware antes de passar pela CPU. Porém, eles precisam condensar os dados filtrados em páginas para evitar a poluição do bufferpool que é custoso e propenso a erros. Por outro lado, os protocolos atuais de memória lidam com todas as idiossincrasias de instruções PIM, tais como: coerência de cache, correção de erros de códigos de memória (ECC) e acesso direto à memória (DMA). O fluxo de execução funciona no nível de instrução como o processamento tradicional da CPU (e.g., AVX/SSE): os programadores adicionam instruções PIM intrínsecas, como as Intel Intrinsics, e o compilador sinaliza-as como instruções de memória especiais.

\section{Processamento de Consulta com PIM}

Para avançarmos no desenvolvimento do processamento de consulta PIM-consciente, precisamos entender o impacto do uso do PIM na execução de consulta tradicional. Por isso, analisamos tal impacto em uma carga de trabalho analítica ao comutar o processamento entre CPU e PIM em termos do tempo de execução e consumo de energia dos operadores de projeção, seleção e junção. Realizamos nossa análise através do simulador SiNUCA devido às limitações de hardware e usamos os mesmos parâmetros aplicados por trabalhos relacionados [Tome and et al. 2018] e estimamos o consumo de energia com os mesmos parâmetros de DRAM do estado da arte [Jeddeloh and Keeth 2012]. Além de tudo, o SiNUCA tem sido extensivamente adotado em artigos científicos em arquitetura de computadores [Alves and et al. 2016] e banco de dados [Tome et al. 2018, Kepe 2018].

Avaliamos os operadores através do benchmark TPC-H de 1GB porque os dados de entrada cabem em cache que é o cenário mais propício para processamento em CPU. As implementações dos operadores usam operações SIMD de 64-bytes (CPU/AVX-512) e 256-bytes (PIM). Os resultados convergem para explorarmos nossa visão de SGBD-PIM.
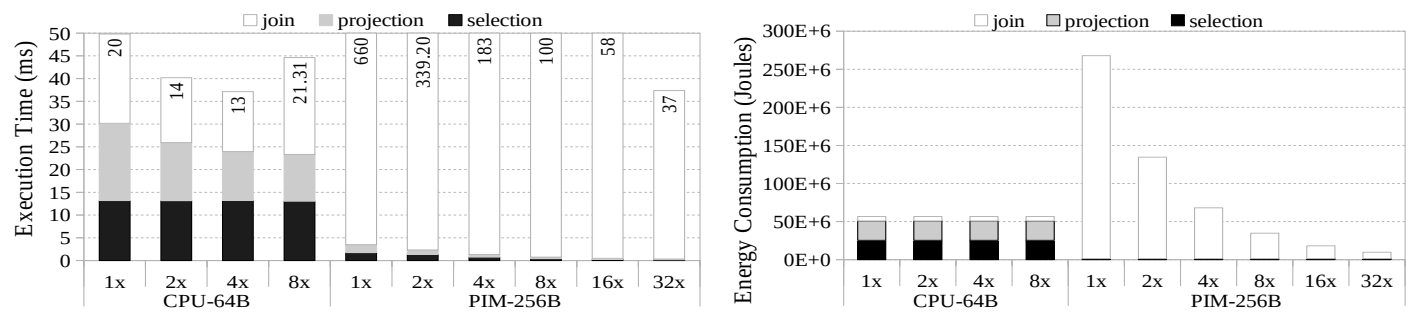

Figura 2. Tempo de execução e consumo de energia dos operadores da consulta 03 do TPC-H: seleção, projeção e junção em CPU-64B e PIM-256B com profundidades de loop desenrolados entre $1 \times$ até $32 \times$.

Projeção: A Figura 2 mostra que a projeção em PIM reduz o tempo de execução em mais de uma ordem de magnitude, cerca de $60 \times$. Também observamos uma redução de consumo de energia em 36×. Seleção: A seleção com PIM reduz o consumo de energia em $98 \%$ e o tempo de execução em 76× comparado a execução em CPU. 
Os resultados são devidos ao comportamento em streaming dos operadores com PIM que causa baixo reuso de dados e pouca movimentação destes fora da memória principal. No processamento em CPU, as colunas são transferidas desde a memória principal passando por toda hierarquia de caches até a CPU que compara porções das colunas com os predicados de seleção e projeção. Contudo, todas as porções de dados transferidas não são reutilizadas durante a execução, inibindo o mecanismo de caching. Em contrapartida, nos operadores em PIM, a CPU envia apenas as instruções para o dispositivo PIM. Os operadores acessam e avaliam os dados com até 32 instruções PIM+SIMD de 256-bytes.

Junção: Em nossos experimentos, implementamos três algoritmos de junção, porém os algoritmos de Hash Join e Sort-Merge Join geram acessos aleatórios à memória que inibem o acesso paralelo aos dados e a alta largura de banda dos dispositivos PIM. Por isso, para fornecer uma percepção sobre os problemas de junção em PIM, nós analisamos a execução do Nested Loop Join (NLJ). O NLJ-PIM desenrola o loop interno até $32 \times$ para explorar os acessos paralelos aos dados. Porém, cada iteração desse loop causa instruções compulsórias de leitura e escrita na memória, pois a coluna de junção interna (inner column em cache) é reacessada em toda iteração do loop. Por isso, o processamento em CPU é melhor que a execução em PIM, chegando a ser 2,8× mais rápido, conforme mostra o gráfico da Figura 2. Mesmo o consumo de energia, o maior benefício de usar PIM, é 70\% mais custoso em PIM. Entretanto, o NLJ-PIM torna-se viável quando a coluna interna de junção não cabe em cache o que inibe o reuso de dados. Neste ponto, o PIM obtém uma melhora de 1,38× comparado ao processamento em CPU.

\section{Processamento de Consulta PIM-Consciente}

Em nosso estudo preliminar sobre o impacto de PIM no processamento consulta, nós observamos que nem todo operador de banco de dados se beneficia do PIM. Portanto, a decisão sobre a arquitetura de processamento de um operador de consulta não é trivial. De fato, observamos que os operadores de consulta com alta reutilização de dados se beneficiam do mecanismo de caching e, assim, o processamento em CPU torna-se atraente, como no caso do NLJ. Por outro lado, os operadores com comportamento streaming (e.g., projeções e seleções) são mais adequados para o PIM. Outros operadores, como a agregação, podem ter um reuso de dados baixo ou médio e um comportamento de streaming parcial, o que torna pouco evidente qual é o melhor dispositivo de processamento.

Nossa investigação concentra-se em como intercalar a execução de consultas entre a CPU e o PIM, mas entendemos que um otimizador de planos PIM-consciente deve ser averiguado. Iniciamos nosso estudo com um escalonamento estático que usa um modelo de classificação baseado no perfil de operadores para decidir em qual arquitetura processar um dado operador. Diferente de trabalhos relacionados em escalonamento para GPU, esta estratégia não requer uma fase de calibração [Breß et al. 2012] nem heurística [Karnagel et al. 2014]. A Figura 3 apresenta três planos de execução de consulta a partir do plano ótimo: dois planos hardware-específicos, i.e., CPU e PIM, e um plano híbrido baseado no escalonamento estático de perfil. No plano híbrido, os operadores de seleção e projeção são processados em PIM, enquanto a junção é executada em CPU. Com o plano híbrido a execução de consulta tem uma melhora em quase $3 \times$ (Figura $3 \mathrm{~d}$ ), pois explora as vantagens de ambas as arquiteturas: operadores com alto reuso de dados são executados na CPU e operadores streaming são processados em PIM. Em termos de consumo de energia, o plano híbrido chega a reduzir em pelo menos $25 \%$, Figura $3 \mathrm{e}$. 


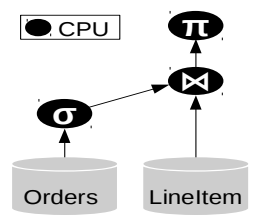

(a) CPU Plan.

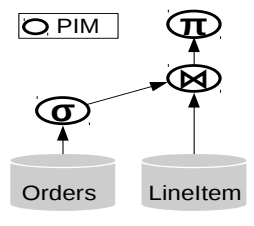

(b) PIM Plan.

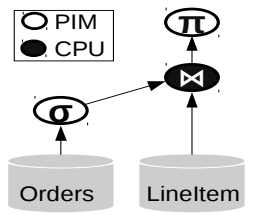

(c) Hybrid Plan.

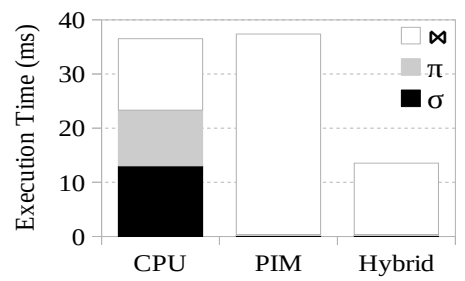

(d) Execution Time.

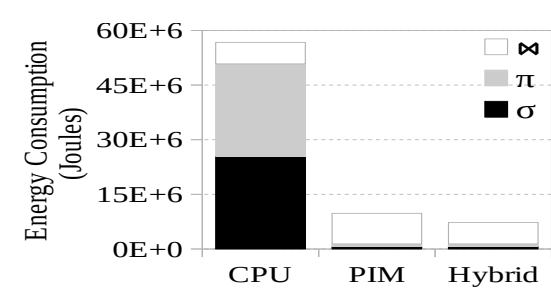

(e) Energy Consumption.

Figura 3. Planos de consulta: planos isolados CPU (a) e PIM (b), plano híbrido

(c). Respectivos tempo de execução (d) e consumo de energia (e).

\section{Desafios \& Oportunidades}

Coprocessamento Simultâneo: Em ambientes de processamento heterogêneos, o otimizador precisa identificar oportunidades de coprocessamento para evitar dispositivos ociosos ou consumo de energia ineficiente (e.g., o problema do muro de energia [Wang and Skadron 2013]). Além disso, a detecção de concorrência à memória é difícil de gerenciar no processamento simultâneo entre CPU e PIM.

Otimização do Plano de Consulta: O espaço de busca na otimização de planos de consulta já é um problema comum ao otimizador de consulta. A adição de planos híbridos aumenta ainda mais a complexidade para gerar planos de consultas candidatos. Desta forma, um otimizador de consulta PIM-consciente deve considerar características específicas de hardware para escolher o dispositivo de processamento apropriado.

Transações: Intrinsecamente, as instruções PIM aritméticas e lógicas são atômicas [HMC-Consortium 2015], o que proporciona oportunidades de pesquisa para transações em PIM e Hybrid Transactional/Analytical Processing (HTAP). A ISA atual dos dispositivos PIM suportam instruções do tipo comparação-e-troca para teste de dados. Por conseguinte, instruções de atualização PIM podem ser sincronizadas em memória sem necessitar de checar as caches ou uso extra da banda de memória. A oportunidade é reduzir o overhead de bloqueio de dados e de estruturas (locking e latching) que correspondem a 30\% das instruções OLTP [Harizopoulos and et al. 2008].

Adoção do SGBD: Em nossa visão SGBDs devem invocar instruções PIM no código dos operadores, similarmente as abordagens SSE e AVX. Nós também consideramos otimização de código para incluir funções intrínsecas na ISA dos dispositivos PIM.

\section{Conclusão \& Trabalho Futuro}

Neste artigo apresentamos nossa visão de um SGBD que explora a largura de banda de memória sem precedentes dos novos dispositivos PIM aliada ao processamento em memória. Discutimos resultados promissores ao intercalar a execução de processamento intra-consulta entre PIM e CPU. Com nosso escalonador estático, planos de consulta 
híbridos melhoraram o desempenho cerca de $3 \times$ e reduziram o consumo de energia em cerca de $25 \%$. Apesar desses resultados promissores, sabemos que existem limitações a serem superadas. Por isso, nosso trabalho em andamento consiste em um escalonador dinâmico para criar e atualizar perfis e reescalonar operadores dinamicamente. Também consideramos investigar o otimizador de consulta PIM-consciente e os algoritmos Hash Join, Sort-Merge Join e agregações. Por último, compartilhamos com a comunidade uma lista de desafios e oportunidades abertas pela nossa visão para o co-design do SGBD-PIM.

AGRADECIMENTOS. Este trabalho foi parcialmente financiado pelo Instituto Serrapilheira (número de concessão Serra-1709-16621).

\section{Referências}

Alves, M. A. Z. and et al. (2016). Large vector extensions inside the HMC. In DATE.

Breß, S., Mohammad, S., and Schallehn, E. (2012). Self-tuning distribution of dboperations on hybrid CPU/GPU platforms. In 24th Grundlagen von Datenbanken.

Do, J., Kee, Y., Patel, J. M., Park, C., Park, K., and DeWitt, D. J. (2013). Query processing on smart ssds: opportunities and challenges. In SIGMOD.

Harizopoulos, S. and et al. (2008). OLTP through the looking glass, and what we found there. In SIGMOD.

HMC-Consortium (2015). Hybrid Memory Cube Specification 2.1. HMC-30G-VSR PHY.

Jeddeloh, J. and Keeth, B. (2012). Hybrid memory cube new DRAM architecture increases density and performance. In VLSIT.

Karnagel, T., Habich, D., Schlegel, B., and Lehner, W. (2014). Heterogeneity-aware operator placement in column-store DBMS. Datenbank-Spektrum.

Kautz, W. H. (1969). Cellular logic-in-memory arrays. IEEE Trans. Computers.

Keeton, K. and et al. (1998). A case for intelligent disks (idisks). SIGMOD Record.

Kepe, T. R. (2018). Dynamic database operator scheduling for processing-in-memory. In PhD@VLDB.

Kim, J. and Kim, Y. (2014). HBM: memory solution for bandwidth-hungry processors. In 26th HCS.

Mirzadeh, N., Kocberber, O., Falsafi, B., and Grot, B. (2015). Sort vs. hash join revisited for near-memory execution. In ASBD@ISCA.

Patterson, D. A. and et al. (1997). A case for intelligent RAM. IEEE Micro.

Tome, D. G. and et al. (2018). HIPE: HMC instruction predication extension applied on database processing. In DATE.

Tome, D. G., Kepe, T. R., Alves, M. A. Z., and de Almeida, E. C. (2018). Near-data filters: Taking another brick from the memory wall. In ADMS@VLDB.

Wang, L. and Skadron, K. (2013). Implications of the power wall: Dim cores and reconfigurable logic. IEEE Micro. 